\title{
Astrometric optical interferometry with non-evacuated delay lines
}

\author{
G. Daigne ${ }^{1}$ and J.-F. Lestrade ${ }^{2}$ \\ 1 Observatoire de Bordeaux, BP. 89, F-33270 Floirac, France \\ e-mail: daigne@observ.u-bordeaux.fr \\ 2 Dept. Arpèges, Observatoire de Paris-Meudon, F-92191 Meudon Cedex, France \\ e-mail: lestrade@obspm.fr
}

Received April 9; accepted June 8, 1999

\begin{abstract}
The effects of longitudinal dispersion in long baseline optical interferometry with non-evacuated delay lines are analyzed. A new technique is proposed for group delay tracking, with phase measurements in two wavebands only. Sensitivity and instrumental uncertainties are further investigated, together with the dependence on stellar color temperature. To a precision level of a few $10^{-10}$ rad, limiting factors in dual-field astrometry should be put under control with not too long interferometer baseline.
\end{abstract}

Key words: athmospheric effects - techniques: interferometric - astromety

\section{Introduction}

Long-baseline optical interferometry do have a potential for sub-mas precision in astrometric measurements as first suggested by Shao \& Staelin (1977). Furthermore, with the dual-field technique and simultaneous observation of two nearby sources, long time averaging of differential path length fluctuations makes it achievable precision at a $10-20 \mu$ as level (Shao \& Colavita 1992). This is the range of precision required for the detection of Jupiterlike extrasolar planets through the reflex motion of their central star, projected onto the celestial sphere; e.g. the amplitude of the reflex motion of the sun at 10 parsec is 0.5 mas.

Astrometric interferometers for wide-angle measurements, MkIII (Shao et al. 1988) and Navy Prototype Optical Interferometer or NPOI (Armstrong et al. 1998), have their main delay lines located in evacuated pipes. Zeroing the residual phase of the white-fringe holds the scales between an outside geometric delay depending on the source position in the sky, and the compensating vacuum delay within the interferometer arms. With

Send offprint requests to: G. Daigne non-evacuated delay lines, longitudinal dispersion has to be considered: the relation between residual phase and wavenumber is no longer linear, and there is no longer any white-fringe.

After the first implementation of a dual-field interferometer, the Palomar Testbed Interferometer or PTI (Colavita et al. 1994; Colavita et al. 1999), unique astrometric results appear to be on the reach. The Very Large Telescope Interferometer (VLTI) at Paranal will have all its optical paths along non-evacuated pipes (von der Lühe et al. 1994; Mariotti et al. 1998). Although residual air turbulence is not expected to be a limiting factor in its operation (Koehler et al. 1997), longitudinal dispersion effects have to be considered (Lévêque 1997). Although this instrument is not supposed to work as an astrometric interferometer for wide-angle measurements, a dual-field instrument for narrow-angle astrometry is being studied (Quirrenbach et al. 1998) and some wide-angle astrometry is needed, at least for baseline calibration.

With an astrometric interferometer, fringe tracking is not just trying to keep the residual delay small enough for the visibility remaining near its maximum value. The residual delay has to be accurately measured, as well as the optical path and/or group delay introduced by the delay line. With non-evacuated pipes, these last quantities are wavelength dependent, and the metrology has to be scaled to some effective wavelength really observed. In this paper, we shall examine the operation of an astrometric interferometer in the near-IR range and draw some inferences on the design of a fringe sensor unit, in terms of its sensitivity and dependence on ambient parameters. The scheme proposed for coherence tracking is the simplest form of group-delay tracking or GDT (Lawson 1995), with the observation of two wavebands only. It has not to be confused with the two-color technique developed in the visible range and used with the MkIII interferometer (Colavita et al. 1987). Indeed, the air dispersion factor is more than 10 times smaller in the near-IR band (between $H$ and $K$ bands), as compared to the visible, so that 
highly precise phase measurements would be required for it to be useful. The two-color technique was implemented with the MkIII interferometer, for partial correction of the atmospheric path length fluctuations ahead. A two-bands group-delay tracking is here investigated to get rid of longitudinal dispersion along the non-evacuated delay lines of an IR astrometric interferometer.

A dual-field interferometer is a kind of a two-stages apparatus: the uncertainty on small angle measurements is the product of the wide-angle, or single-field astrometric accuracy, by the separation angle to be measured, expressed in radian. The single-field accuracy is about the same as the relative uncertainty on the interferometer baseline. For angles as small as a few arcminutes, or $10^{-3}$, and error on relative baseline of about $10^{-7}$, differential astrometry can be performed with a $10^{-10}$ uncertainty, or $20 \mu$ as. These are the figures to be kept in mind for performance estimates.

We first point out the difference between optical path and group delay within interferometer arms with nonevacuated lines. A simple and operating model is proposed for longitudinal dispersion. Group-delay tracking and the effects of stellar emission spectra (or color temperature) on astrometric measurements are developed in Sect. 3 . Sensitivity and performances of a proposed concept for fringe tracking are further analyzed.

\section{Optical length and group delay}

The atmosphere is first supposed to be a stratified medium without turbulence. The baseline $\boldsymbol{B}$ is horizontal, and much shorter than the scale height of the atmosphere. Let $n(\sigma)$ the refractive index of the atmosphere at the interferometer level, and $\sigma$ the wavenumber or inverse of the wavelength, $s(\sigma)$ the source direction as seen by the instrument. The Optical Path Delay of the rays at the two entrance pupils, or geometrical $O P D$, is the scalar product $n(\sigma) \boldsymbol{B} \cdot \boldsymbol{s}(\sigma)$. With the Snell's law on ray propagation, this quantity keeps to be the same all along the ray path through the atmosphere, and it equals its value outside the atmosphere:

$O P D_{\text {geo }}=\boldsymbol{B} \cdot \boldsymbol{s}_{\mathbf{0}}$

where $\boldsymbol{s}_{\mathbf{0}}$ is the source direction outside the atmosphere, or without refraction. The quantity $O P D_{\text {geo }}$ is also the geometric group delay for the propagation of wavepackets.

For wave propagation through an isotropic dispersive medium, two features have to be considered:

- propagation of lateral coherence, or of wave surfaces normal to the ray paths. The phase variation between two points $P_{1}$ and $P_{2}$ along a ray path is proportional to the optical length $L_{\phi}(\sigma)$ between this two points with:

$L_{\phi}(\sigma)=\int_{P_{1}}^{P_{2}} n(\sigma) \mathrm{d} s$
- propagation of longitudinal coherence, or of wavepackets. Between two points $P_{1}$ and $P_{2}$ along a ray path, the group delay $L_{\mathrm{g}}(\sigma)$ is:

$L_{\mathrm{g}}(\sigma)=\int_{P_{1}}^{P_{2}} \frac{c}{v_{\mathrm{g}}(\sigma)} \mathrm{d} s$

where $v_{\mathrm{g}}$ is the group velocity:

$v_{\mathrm{g}}=\frac{c}{n(\sigma)}\left[1-\frac{\sigma}{n(\sigma)} \frac{\partial n}{\partial \sigma}\right]$

In a weakly dispersive medium such as the air, we shall have:

$\frac{c}{v_{\mathrm{g}}(\sigma)} \simeq n(\sigma)\left[1+\frac{\sigma}{n(\sigma)} \frac{\partial n}{\partial \sigma}\right]$

and in the visible and near-IR range, the difference between optical length and group delay is as small as a few parts in $10^{6}$. Nevertheless the use of $O P D$ for the whole optical path within the interferometer with non-evacuated pipes is ambiguous. Does it stand for optical length or for group delay? so that we shall use it only for the geometrical delay outside the interferometer, eventually affected by the fluctuations of the atmospheric piston:

$O P D=O P D_{\text {geo }}+\epsilon_{\text {atmos }}$

the long term average of the last quantity being zero.

\subsection{Evacuated delay line}

With an evacuated delay line with length $L_{\text {del }}$, the basic equation for the residual optical length of the interferometer is:

$\Delta L_{\phi}=O P D-L_{\mathrm{del}}+C_{\mathrm{off}}$.

As long as the interferometer "offset" $C_{\text {off }}$ is achromatic or non dispersive, there exists a fringe with zero phase at any wavenumber. The "white-fringe" is obtained for $\Delta L_{\phi}=0$. This is also the condition for zero group delay, so that the maximum coherence is obtained at the whitefringe, and all these are well-known results for an optical interferometer with evacuated delay line.

\subsection{Non-evacuated delay line}

The interferometer offset will not be further considered in this paper, and dropped to zero in the following. A delay line with length $L_{\text {del }}$ located in an air-filled tunnel will compensate exactly the $O P D$ at a single wavelength only. Furthermore two types of compensation are obtained, either optical length compensation or group delay compensation, the residual lengths being respectively given by $\Delta L_{\phi}$ and $\Delta L_{\mathrm{g}}$ with:

$\Delta L_{\phi}(\sigma)=O P D-n(\sigma) L_{\mathrm{del}}$
$\Delta L_{\mathrm{g}}(\sigma)=O P D-\frac{c}{v_{\mathrm{g}}(\sigma)} L_{\mathrm{del}}$ 
and with (5):

$\Delta L_{\mathrm{g}}(\sigma)=O P D-\left[n(\sigma)+\sigma \frac{\partial n}{\partial \sigma}\right] L_{\mathrm{del}}$.

The residual phase being $\phi=2 \pi \sigma \Delta L_{\phi}$, the relation between residual phase derivative and residual group delay is easily checked:

$\frac{1}{2 \pi} \frac{\mathrm{d} \phi}{\mathrm{d} \sigma}=\Delta L_{\mathrm{g}}(\sigma)$.

Coherencing, or group delay tracking with $\Delta L_{\mathrm{g}}(\sigma)=0$, has to be performed at a peculiar wavenumber determined by the instrumental set-up. Let $\sigma_{0}$ its value, and $\sigma_{0}^{\prime}$ the wavenumber with zero residual phase, it is solution of:

$n\left(\sigma_{0}^{\prime}\right)=n\left(\sigma_{0}\right)+\left.\sigma_{0} \frac{\partial n}{\partial \sigma}\right|_{\sigma_{0}}$.

The air refractive index being an increasing function wrt wavenumber, $\sigma_{0}^{\prime}>\sigma_{0}$.

\subsection{A model for the air refractive index}

There is no simple analytical form for the air refractive index in a wide optical range. Fits to index measurements lead to the following general expression in terms of the wavenumber $\sigma$ (see e.g. Owens 1967):

$n(\sigma)-1=A+\frac{B}{C-\sigma^{2}}+\frac{D}{E-\sigma^{2}}$

where the coefficients are dependent on the partial pressures of the minor components (water vapor, carbon dioxyde, ...), and on temperature and total pressure.

In the near-IR range, with $\sigma<1 \mu \mathrm{m}^{-1}$, only a few terms in a $\sigma^{2}$ power expansion of the preceeding expression are to be considered. With the additional assumption of the dry component and wet air component obeying the ideal gas law, both separately and for the combined mixture, a dry air refractive index expansion is given by Gubler \& Tytler (1998):

$n(\sigma)-1=\left(A_{0}+B_{0} \sigma^{2}+C_{0} \sigma^{4}\right) \frac{T_{0}}{T} \frac{p}{p_{0}} \cdot 10^{-6}$

with $A_{0}=287.604, B_{0}=1.6288, C_{0}=0.0136$, and standard conditions $T_{0}=273.15 \mathrm{~K}$ and $p_{0}=1013.25 \mathrm{hPa}$.

In this paper, the numerical applications will be performed for the atmospheric conditions prevailing in the VLTI interferometer tunnel, i.e. $T=289 \mathrm{~K}$ and $p=$ $743 \mathrm{hPa}$ (Lévêque 1997), so that (14) reduces to:

$n(\sigma)=1+\alpha+\beta \sigma^{2}+\gamma \sigma^{4}$

with $\alpha \simeq 199.32910^{-6}, \beta \simeq 1.12910^{-6} \mu \mathrm{m}^{2}$ and $\gamma \simeq$ $0.00910^{-6} \mu \mathrm{m}^{4}$.

To better than a few parts in $10^{3}$ and for better clarity, we shall neglect the $\sigma^{4}$ term in the analytic developments of the air dispersive effect. The contribution of water vapor to dispersion effects may be difficult to modelize in the near-IR transmission bands, that is in the vicinity of water vapor absorption bands. With the conditions prevailing in the VLTI tunnel, its contribution, although small, is not known exactly and should be measured and monitored during astrometric observations.
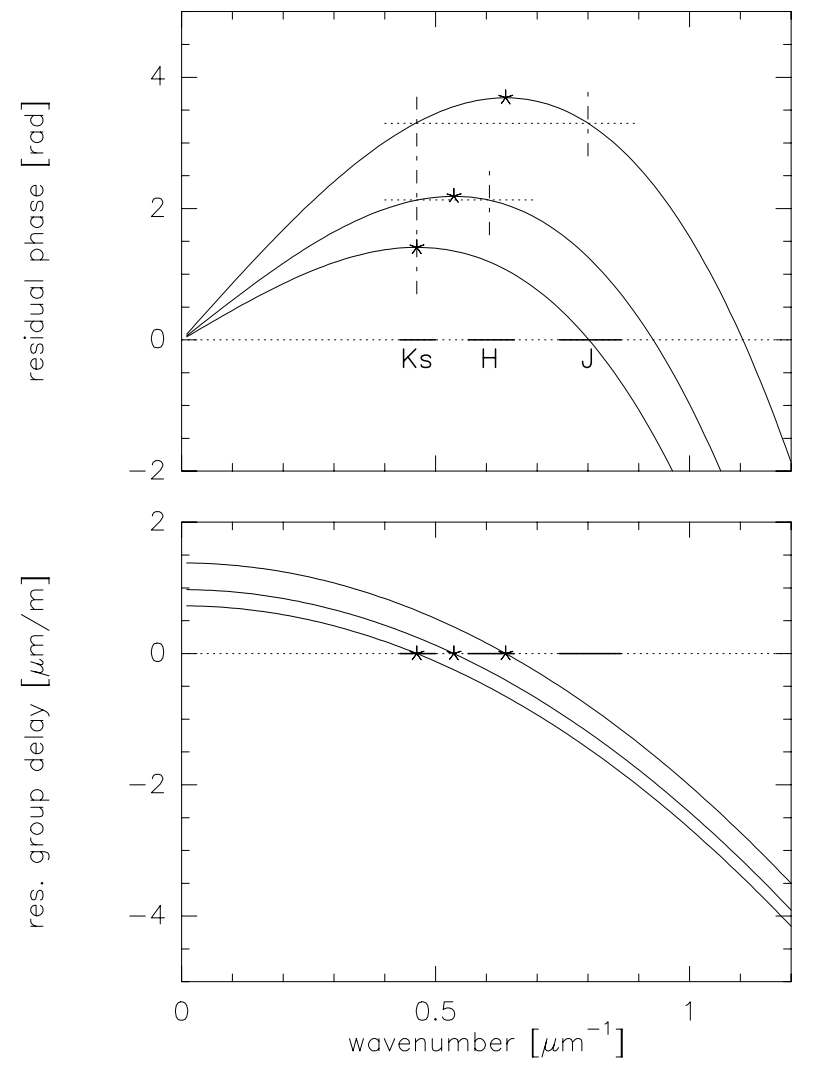

Fig. 1. Residual phase and residual group delay per meter of air-filled delay line, and for the 3 pairs of guiding wavenumbers (see Table 1). Zero group delay is shown as a $\left(^{*}\right)$ on each curve. The near-IR wavebands with $15 \%$ relative width are also shown (full segments)

\subsection{The dispersive term}

The departure to a white-fringe, with zero residual phase and group delay, is due to the $\beta \sigma^{2}$ term in the simplified expression for the refractive index, and the solution to (12) is:

$\sigma_{0}^{\prime} \simeq \sqrt{3} \sigma_{0}$.

The residual phase is an odd cubic function of the wavenumber given by:

$\phi(\sigma) \simeq 2 \pi \sigma\left(3 \sigma_{0}^{2}-\sigma^{2}\right) \beta L_{\mathrm{del}}$

with its maximum at $\sigma=\sigma_{0}$. Equal phase can be measured at two different wavenumbers $\sigma_{1}$ and $\sigma_{2}$ with $\sigma_{1}<$ $\sigma_{0}<\sigma_{2}$, and this property will be used in the proposed fringe tracking system. Residual phase is plotted in Fig. 1 for 1 meter of delay line, and for three different values of $\sigma_{0}$, ranging from the $K$ band to the $H$ band.

The residual group delay is:

$\Delta L_{\mathrm{g}}(\sigma) \simeq 3\left(\sigma_{0}^{2}-\sigma^{2}\right) \beta L_{\mathrm{del}}$

that is $-6 \sigma_{0}^{2} \beta L_{\mathrm{del}}$ for zero residual phase, or a few $\mu \mathrm{m}$ per meter of delay line. The residual group delay is plotted in Fig. 1 for the three different $\sigma_{0}$ values. It is seen also as the slope of the phase curves. 


\section{A fringe tracking unit with air-filled delay line}

\subsection{Coherencing operation}

A fringe tracking unit is needed in order to reduce the $O P D$ variations, either systematic or random, to a fraction of the coherence length. The delay line is permanently adjusted for this purpose, and its motion is controlled by the error signal of the tracking unit. Simultaneous observations in at least two different wavebands are needed. Let $\sigma_{1}$ and $\sigma_{2}$ the mean wavenumbers of the two bands, that we call guiding wavenumbers. The phase difference $\Delta \phi_{12}=\phi\left(\sigma_{2}\right)-\phi\left(\sigma_{1}\right)$ is, with $(17)$ and $\Delta \sigma_{12}=\sigma_{2}-\sigma_{1}$ : $\Delta \phi_{12} \simeq 2 \pi \Delta \sigma_{12}\left[3 \sigma_{0}^{2}-\left(\sigma_{1}^{2}+\sigma_{1} \sigma_{2}+\sigma_{2}^{2}\right)\right] \beta L_{\mathrm{del}}$.

Maintaining zero phase difference whatever the delay length gives the condition for zero group delay tracking at $\sigma_{0}$ :

$\sigma_{0}^{2}=\frac{1}{3}\left(\sigma_{1}^{2}+\sigma_{1} \sigma_{2}+\sigma_{2}^{2}\right)$

The wavenumber with zero group delay has to be precisely known. As it stands at the residual phase maximum, $\sigma_{0}$ is further called leading wavenumber. Indeed, laser metrology of the delay line has to be corrected for air longitudinal dispersion, either at $\sigma_{0}$ for group delay metrology, with two colors, or at $\sigma_{0}^{\prime}$ for optical length or phase measurements, with a single color. These two wavenumbers are approximately given by (20) and (16), to a few parts in $10^{3}$. More accurate expressions would be obtained with the next term in the power expansion of the refractive index.

\subsection{Choice of the guiding wavenumbers}

The two observed bands should be large enough for sensitivity purposes, and with a convenient separation: not too close for a good sensitivity to delay variations, and not too far away in order to easily solve for the ambiguity in phase difference. The pair $H$ and $K$ bands seems to be a good compromise, or more precisely the $K_{\mathrm{s}}$ band for reduced thermal contribution.

With $\sigma_{1}=\sigma_{K_{\mathrm{s}}}$, and $\sigma_{2}=\sigma_{H}$, respectively 0.463 and $0.606 \mu \mathrm{m}^{-1}$, the leading wavenumber will be:

$\sigma_{0}=0.536 \mu \mathrm{m}^{-1}$, or $\lambda_{0}=1.865 \mu \mathrm{m}$.

The ambiguity in the phase difference and the sensitivity to delay variations are both given by the relation between phase difference and additional path length $\delta L$ :

$\delta \phi_{12}=2 \pi \Delta \sigma_{12} \delta L$.

With $\Delta \sigma_{12}=0.143$, we obtain a $2 \pi$ variation for $\delta L \simeq$ $7 \mu \mathrm{m}$, and a phase sensitivity of about 1 degree per $20 \mathrm{~nm}$.

Fringe patterns are shown in Fig. 2, for four different values of the delay length $L$, ranging from 0 to $30 \mathrm{~m}$. The amplitude or pattern envelope is here solely determined by the coherence due to the width and shape

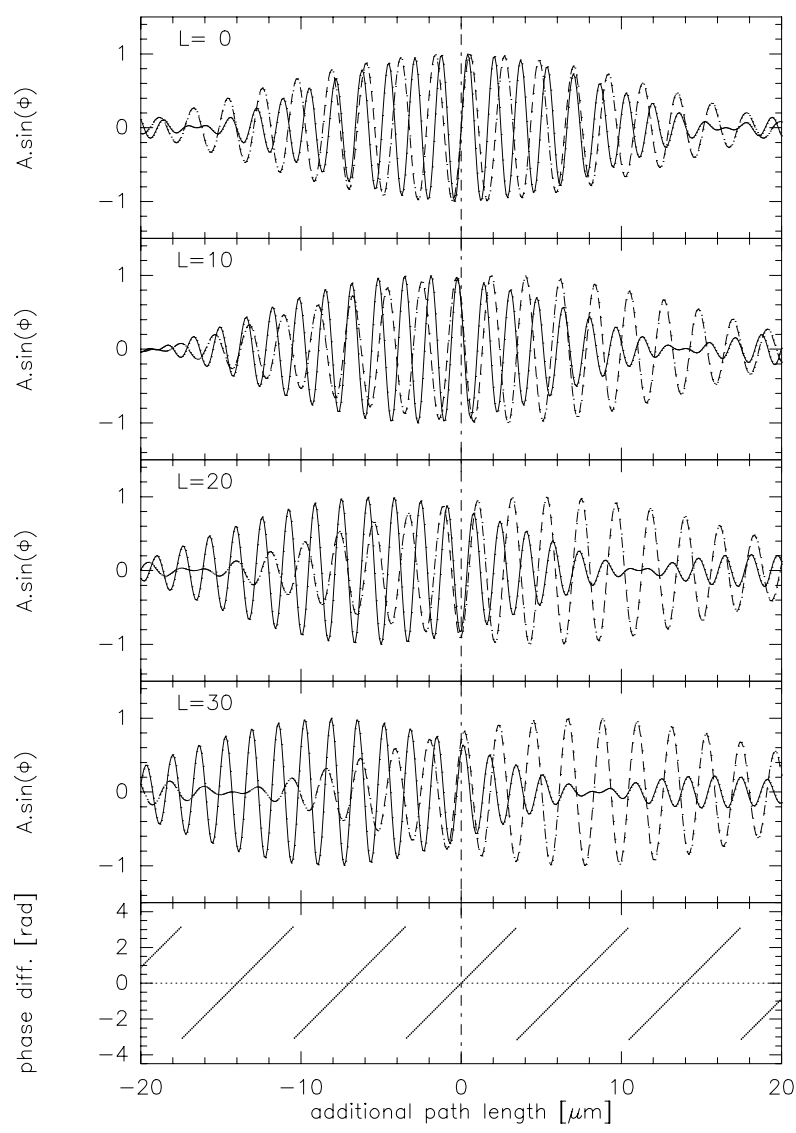

Fig. 2. Sine fringe patterns in the $H$ and $K_{\mathrm{s}}$ bands (full and dashed line respectively) for different air delay length $L$, from 0 to $30 \mathrm{~m}$. The relative bandwidth is 0.1 , with square-shaped band filter. The phase difference $\left(\phi_{H}-\phi_{K_{\mathrm{S}}}\right)$ also shown keeps to be the same whatever the delay length

of the bandpath filters in each observed bands, and the source emission spectrum is supposed to be flat. With a square-shaped bandpath filter of width $\Delta \lambda$, centered at $\lambda=1 / \sigma$, and with resolution $R=\lambda / \Delta \lambda$, the coherence is $\gamma_{\mathrm{c}}(l)=\operatorname{sinc}\left(\pi \sigma_{i} l / R_{i}\right)$ with $i=H$ or $K_{\mathrm{s}}, l$ being the additional delay relative to zero group delay, in each band. The relative bandwidth at the two observed wavebands is supposed to be 0.1 , or $R=10$. The effect of longitudinal dispersion is clearly seen on the position of maximum coherence, shifted on either side of zero delay.

Another choice could be the $J$ and $K_{\mathrm{s}}$ bands. With $\sigma_{1}=\sigma_{K_{\mathrm{s}}}$, and $\sigma_{2}=\sigma_{J}$, respectively 0.463 and $0.80 \mu \mathrm{m}^{-1}$, the leading wavenumber being: $\sigma_{0}=0.638 \mu \mathrm{m}^{-1}$, or $\lambda_{0}=1.565 \mu \mathrm{m}$, that is within the $H$ band. With $\Delta \sigma_{12}=$ 0.337 , a $2 \pi$ variation in phase difference is obtained for $\delta L \simeq 3 \mu \mathrm{m}$, and a sensitivity of about 1 phase degree per $8 \mathrm{~nm}$. The phase ambiguity is here more critical, but this could be solved with a third band observation, for example in the $H$ band.

A third choice would be the two guiding wavenumbers in the same near-IR band, say the $K_{\mathrm{s}}$ band. The difference between the two wavenumbers cannot exceed 
Table 1. Residual group delay $\Delta L_{\mathrm{g}}$ in 4 IR bands per meter of delay length, and air delay length $L_{95}$ responsible for $5 \%$ coherence loss with 0.1 relative bandwidth. The different choices investigated for the guiding pairs $\left(\sigma_{1}, \sigma_{2}\right)$ are shown

\begin{tabular}{lcclll}
\hline IR band & $J$ & $H$ & $K_{\mathrm{s}}$ & $M$ \\
\hline \multicolumn{2}{l}{ wavelength $[\mu \mathrm{m}]$} & 1.25 & 1.65 & 2.16 & 10.2 \\
\multicolumn{2}{l}{ wavenumber } & 0.800 & 0.606 & 0.463 & 0.098 \\
\hline$\Delta L_{\mathrm{g}}[\mu \mathrm{m}]$ & $\left(K_{\mathrm{s}}, K_{\mathrm{s}}\right)$ & -1.443 & -0.518 & 0.0 & 0.694 \\
& $\left(K_{\mathrm{s}}, H\right)$ & -1.196 & -0.271 & 0.247 & 0.941 \\
& $\left(K_{\mathrm{s}}, J\right)$ & -0.790 & 0.136 & 0.635 & 1.347 \\
\hline$L_{95}[\mathrm{~m}]$ & $\left(K_{\mathrm{s}}, K_{\mathrm{s}}\right)$ & 1.52 & 5.57 & $\infty$ & 25.72 \\
& $\left(K_{\mathrm{s}}, H\right)$ & 1.83 & 10.7 & 15.3 & 18.97 \\
& $\left(K_{\mathrm{s}}, J\right)$ & 2.77 & 21.25 & 5.79 & 13.24 \\
\hline
\end{tabular}

about $0.05 \mu \mathrm{m}^{-1}$, so that a $2 \pi$ variation is reached for $\delta L \simeq 20 \mu \mathrm{m}$, and the sensitivity is about 1 phase degree per $62 \mathrm{~nm}$. This last choice can be thought of as a preliminary or complementary setup for finding the fringe pattern, the $\left(K_{\mathrm{s}}, H\right)$ pair being favored for high precision measurements.

\subsection{Coherence and delay range}

The residual group delay (18) is a linear function of the delay length. It is given in Table 1 , for the $J, H, K_{\mathrm{s}}$ and $M$ bands, per meter of delay length at Paranal. It has to be kept much smaller than the coherence length for fringes to be observed, and the effect will be more stringent at shorter wavelength. The coherence is given by $\gamma_{\mathrm{c}}(l)$ where $l$ now stands for the residual group delay $\Delta L_{\mathrm{g}}(\sigma)$. The air delay length $L_{95}$ responsible for a $5 \%$ visibility loss, with relative bandwidth of 0.1 , has been estimated in the 4 bands, and is given in Table 1 . The three just mentioned choices for the guiding wavenumbers have been considered.

\subsection{Compensation of the air dispersion}

Glass compensation plates will be needed with delay lengths larger than about 20 meters and the $\left(K_{\mathrm{s}}, H\right)$ pair for fringe tracking (Fig. 2). The problem has been investigated by Lévêque (1997) for the VLTI delay lines, and the same kind of results is obtained with our approximation for the air dispersive component. Let $N(\sigma)$ the glass refractive index, $N_{0}^{\prime}$ and $N_{0}^{\prime \prime}$ respectively its first and second derivatives wrt wavenumber at $\sigma=\sigma_{0}$. The addition of a glass plate with thickness $e$ in one of the interferometer arms gives a group delay change $E(\sigma)$ :

$E(\sigma) \simeq\left[N(\sigma)-1+\sigma \frac{\partial N}{\partial \sigma}\right] \cdot e$

and the compensation of the group delay dispersion reduces to:

$E(\sigma)-E\left(\sigma_{0}\right)=\Delta L_{\mathrm{g}}(\sigma)$.
In the useful wavenumber interval $\left[\sigma_{1}, \sigma_{2}\right]$, the glass refractive index is supposed to be correctly described by its series expansion, at order 2 :

$N(\sigma) \simeq N\left(\sigma_{0}\right)+\left(\sigma-\sigma_{0}\right) N_{0}^{\prime}+\frac{1}{2}\left(\sigma-\sigma_{0}\right)^{2} N_{0}^{\prime \prime}$.

With (22), it is shown that group delay compensation at $\sigma_{1}$ and $\sigma_{2}$, and then in the whole range $\left[\sigma_{1}, \sigma_{2}\right]$, can be obtained if and only if $N_{0}^{\prime}=\sigma_{0} N_{0}^{\prime \prime}$, that is:

$N(\sigma)=N\left(\sigma_{0}\right)+\frac{1}{2}\left(\sigma^{2}-\sigma_{0}^{2}\right) N_{0}^{\prime \prime}$.

The thickness of the glass plate will be $e=2 \beta L_{\mathrm{del}} / N_{0}^{\prime \prime}$.

The glass plate will add an additional delay at $\sigma_{0}$, given by (22) with $\sigma=\sigma_{0}$, so that the air delay line has to be moved accordingly. This jump has not to be known exactly if we accept a different interferometer offset $C_{\text {off }}$ for each added compensation plate, the offset being determined through astrometric calibration of the instrument, with the observation of reference stars.

\subsection{Effect of a non flat emission spectrum}

With the proposed scheme for fringe tracking and airfilled delay lines, phase measurements are performed in two wavebands at non zero residual group delay. The measured phase $\phi(l)$ is then dependent on the shape of the observed spectrum, and given by:

$\gamma_{\mathrm{c}}(l) \mathrm{e}^{i \phi(l)}=\frac{\int_{\text {band }} g(\sigma) I(\sigma) \mathrm{e}^{2 i \pi \sigma l} \mathrm{~d} \sigma}{\int_{\text {band }} g(\sigma) I(\sigma) \mathrm{d} \sigma}$

where $\gamma_{\mathrm{c}}(l)$ is the module of the coherence factor, $g(\sigma)$ the shape of the bandpass filter (supposed to be squareshaped), and $I(\sigma)$ the source intensity spectrum.

The phase measured in the two guiding bands can be written:

$\phi_{i}\left(l_{i}\right)=2 \pi \sigma_{i} l_{i}+\psi_{i}\left(l_{i}\right)$

with $i=1,2$, and $\psi_{i}\left(l_{i}\right)$ the "spectrum phase", due to the non-flat observed spectrum. The residual delay is $l_{i}=\Delta L_{\mathrm{g}}\left(\sigma_{i}\right)$, given by (18) in terms of the air delay length.

The residual group delay has to be much smaller than the coherence length $1 / \Delta \sigma$ for a large enough coherence module, so that fine structures in the source emission spectrum will have negligeable effects on the residual phase. As we are concerned with astrometric measurements and large bandwidth observations, the observed spectrum is supposed to be characterized by a single parameter, the color temperature $T_{\mathrm{c}}$ of the equivalent black body radiation spectrum:

$I(\sigma) \propto \frac{\sigma^{3}}{\exp (\sigma / \eta)-1}$

with $\eta=6.9410^{-5} T_{\mathrm{c}} \mu \mathrm{m}^{-1}$.

The "spectrum phase" is shown in Fig. 3 in terms of the air delay length for the $K_{\mathrm{s}}$ and $H$ bands, and for different values of the color temperature parameter, ranging 

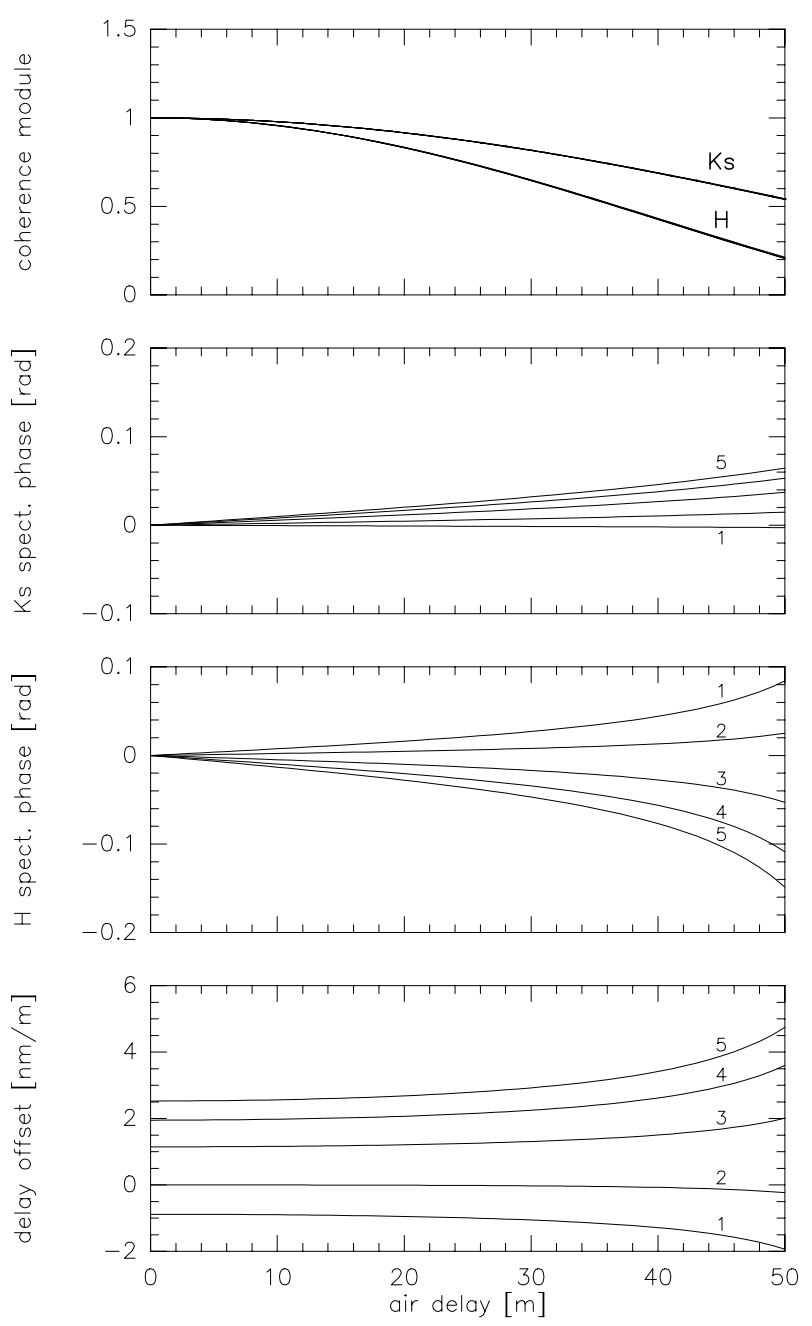

Fig. 3. Effect of the source emission spectrum, characterized by its color temperature $T_{\mathrm{c}}$, on the phase measured in each band, and on the relative delay offset due to the spectral shape. The curve parameter ranging from 1 to 5 is respectively for $T_{\mathrm{c}}=$ $2300 \mathrm{~K}, 2800 \mathrm{~K}, 4000 \mathrm{~K}, 6000 \mathrm{~K}$ and $10000 \mathrm{~K}$

from $2300 \mathrm{~K}$ to $10000 \mathrm{~K}$. The pic of the black body emission spectrum is found at $2.16 \mu \mathrm{m}$ for $T_{\mathrm{c}} \simeq 2365 \mathrm{~K}$, so that the "spectrum phase" is nearly zero in the $K_{\mathrm{s}}$ band for the first curve. In the $H$ band, a similar situation occurs for $T_{\mathrm{c}} \simeq 3095 \mathrm{~K}$, that is in between curves labelled number 2 and 3 . The coherence module does not show any significant dependence on the temperature of the black body spectrum.

For air delay much larger than $L_{95}$, Fig. 3 shows that the spectrum phase deviation is not linear with the delay length, particularly in the $H$ band. The consequence is a dependence of the relative delay offset $\epsilon$ with the delay length:

$\epsilon\left(L_{\mathrm{del}}\right)=\frac{\psi_{2}\left(L_{\mathrm{del}}\right)-\psi_{1}\left(L_{\mathrm{del}}\right)}{2 \pi \Delta \sigma_{12} L_{\mathrm{del}}}$.

This quantity is plotted in Fig. 3 for the different color temperatures as parameter. The curve with $T_{\mathrm{c}}=2800 \mathrm{~K}$

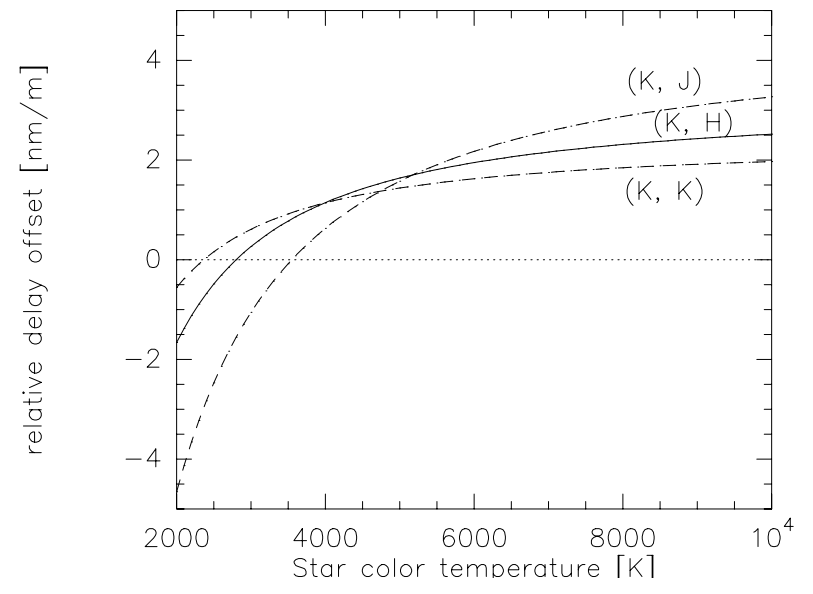

Fig. 4. Relative delay offset due to the non uniformity of the observed spectrum, characterized by its color temperature. Zero delay offset is for a flat spectrum, and the three curves are for the different pairs of guiding wavenumbers (Table 1)

is the less dependent on the delay, as the maximum of the emission spectrum is then located between the two guiding wavenumbers.

Relative to flat emission spectra with guiding wavenumbers $\sigma_{1}$ and $\sigma_{2}$, the mean observed wavenumbers are now $\tilde{\sigma}_{1}$ and $\tilde{\sigma}_{2}$, and the true leading wavenumber $\tilde{\sigma}_{0}$ is now dependent on the source color temperature. Let $\delta \sigma_{0}=\tilde{\sigma}_{0}-\sigma_{0}$. With the derivative of the residual group delay wrt leading wavenumber, the chromatic delay departure is:

$\delta L_{\mathrm{g}} \simeq 6 \sigma_{0} \cdot \delta \sigma_{0} \cdot \beta L_{\mathrm{del}}$

and its relative value $\epsilon_{0}=\delta L_{\mathrm{g}} / L_{\mathrm{del}}$, or relative delay offset for small delay lengths, is plotted in Fig. 4 in terms of the color temperature of the observed source. This departure has no significant impact on the (single-field) astrometric calibration of the interferometer, which is supposed to be at a precision level of a few parts in $10^{8}$, or $1-10$ mas. But for dual-field astrometry and small angle measurements, the situation is different, and $\epsilon_{0}$ gives the chromatic error on the measured angular distance between two sources with different color temperatures. It is particularly significant for stars cooler than about $4000 \mathrm{~K}$. Nevertheless, a precision of a few $10^{-10}$, or about $50 \mu \mathrm{as}$, should be reached with simple modelisation of the emission spectra.

\section{Discussion}

\subsection{Phase measurements}

Phase measurements have to be performed in two wavebands for fringe tracking with air-filled delay lines, and the useful quantity or "observable" is their difference. In each band, phase is continuously changing with the air delay, typically 2 rad per meter, so that the measured phase is 


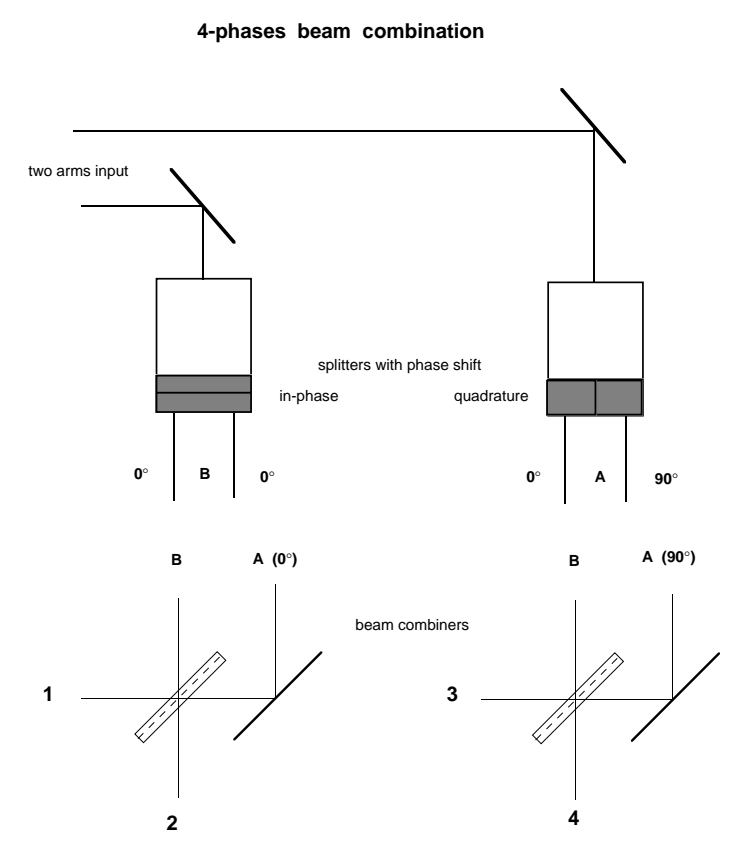

Fig. 5. Basic principle of the two-beam combination in pupil plane, with 4 output phases and wide bands

not constrained. Two schemes can be considered, for beam combination in a pupil plane:

- optical path modulation, at a fast enough rate to freeze the atmospheric turbulence,

- simultaneous in-phase and quadrature-phase measurements.

1) Optical path modulation has been implemented on the first interferometer prototype for optical astrometry (Shao \& Staelin 1980), and later on the MkIII interferometer (Shao et al. 1988), with one wavelength stroke and 4-quadrants measurements. The problem of fringe ambiguity was partly solved with the two-color method which, in the visible, greatly reduced the atmospheric piston effect (Colavita et al. 1987). A more sophisticated technique, with channeled spectrum and delay modulation is implemented on NPOI (Armstrong et al. 1998). This technique gives the fringe amplitude and phase as function of wavenumber. Due to the larger wavelength range of the instrument, about an octave, the stroke is twice the mean wavelength, with 8 samples per scan length. Both with MkIII and NPOI, a single output of the beam combiner is used for fringe measurement. Another approach is to have a 2-quadrants optical path modulation $(0, \lambda / 4)$ with simultaneous measurements of the two complementary outputs of the beam combination (Tango \& Twiss 1980).

2) Simultaneous in-phase and quadrature-phase measurements can be obtained with two combinations as shown in Fig. 5 , and a $\pi / 2$ phase shift in one of the interferometer arm for the quadrature-phase combination. Phase measurements being performed in two different wavebands, the phase shift has to be achromatic which is possible with a polarization splitter and two total reflections in glass material, e.g. an achromatic Fresnel rhomb retarder.

\subsection{Signal/Noise ratio}

We compare the sensitivity for phase measurements in a single band, either with path length modulation and 4quadrants measurements (scheme 1), or with quadraturephase combination (scheme 2). Let $I_{0}$ the incoming source flux, in each interferometer arm, and $T_{0}$ the 4-steps scan duration in scheme 1 or the integration time in scheme 2 . Neglecting instrumental losses, the number of useful photons per measurement and per arm is $n_{0}=I_{0} T_{0}$. With equal intensity splitting before beam combinations, the 4 outputs of Fig. 5 are, in each band:

$J_{k}=\frac{n_{0}}{2}\left[1+\gamma \cos \left(\phi+\theta_{k}\right)\right]$

with $\theta=[-\pi / 2, \pi / 2,0, \pi]$.

The useful quantities for phase measurements are:

$X=J_{1}-J_{2}=\gamma n_{0} \sin \phi$

$Y=J_{3}-J_{4}=\gamma n_{0} \cos \phi$.

Let $n_{\mathrm{b}}$ the background photons number (from a single arm, during $T_{0}$ ), and $r_{\mathrm{n}}^{2}$ the readout noise variance. With Poisson statistic for the photons, the total noise variance per $X$ or $Y$ measurements, each with two reads, is $b^{2}=n_{0}+n_{\mathrm{b}}+2 r_{\mathrm{n}}^{2}$, and the $S N R$ of phase measurements is:

$S N R=\frac{\gamma n_{0}}{\left(n_{0}+n_{\mathrm{b}}+2 r_{\mathrm{n}}^{2}\right)^{1 / 2}}$.

With a 4-steps optical path modulation and a single output in the beam combination (e.g. MkIII), the achievable $S N R$ would be:

$S N R=\frac{\gamma n_{0}}{\sqrt{2}\left(n_{0}+n_{\mathrm{b}}+4 r_{\mathrm{n}}^{2}\right)^{1 / 2}}$

the $\sqrt{2}$ loss factor in the denominator being recovered if the two complementary outputs are simultaneously used. For weak sources, with readout as dominant noise factor, the 4-steps modulation scheme is less sensitive than our proposed scheme, whereas a 2 -steps path modulation scheme $(0, \lambda / 4)$ and detection of the two complementary outputs will give the same figure of merit.

In this sensitivity estimate, we have considered only photon noise and readout noise. In a real situation, visibility fluctuations due to phase corrugations of the wavefront (e.g. Lawson et al. 1999) should be considered as additional atmospheric noise in $S N R$ estimates with optical path modulation. On the other hand, only scintillation noise has to be added in the average $S N R$ estimate of the quadrature-phase scheme, $n_{0}$ being replaced by $n_{0}\left(1+\sigma_{I}^{2}\right)$ in the denominator of (32) where $\sigma_{I}$ is the scintillation index, as seen by the detector. 


\subsection{Longitudinal dispersion and leading wavenumber}

The value of the leading wavenumber is not measured directly, it is deduced from the effective guiding pair $\left(\tilde{\sigma}_{1}, \tilde{\sigma}_{2}\right)$. The relative uncertainty on group delay, and hence on astrometric precision is, with the $\left(K_{\mathrm{s}}, H\right)$ pair and conditions prevailing at Paranal: $\epsilon_{L} \simeq 210^{-6} \epsilon_{\sigma}$ where $\epsilon_{\sigma}$ is the relative uncertainty on the leading wavenumber. That is to say the simple model used here for the air refractive index will be adequate for reaching single-field astrometric precision of a few milliarcseconds, or a few parts in $10^{8}$. For dual-field operations, the uncertainty requirement on differential delay is much more stringent, about one part in $10^{10}$, that is $\epsilon_{\sigma} \leq 10^{-4}$. It means that the light beams from the two observed stars have to cross as much as possible the same dispersive media, band filters, and more generally optical parts. This is why we are investigating a time multiplex scheme for dual-field astrometry, phase measurements in one or the other field being performed without optical path modulation.

With added glass compensation plates, the situation may not be so simple for astrometric calibration of the instrument. Relation (25) for the glass refractive index might not be realized exactly, a consequence being uncontrolled variations of the leading wavenumber $\sigma_{0}$ with delay length.

\subsection{Group delay versus residual phase measurements}

For group delay tracking, the "observable" quantity is a difference in residual phase between two wavebands $\sigma_{1}$ and $\sigma_{2}$, and the residual group delay is vanishing for the leading wavenumber $\sigma_{0}$. In the approximation made for the air refractive index, keeping only the $\sigma^{2}$ term in its development, the $\sigma_{0}$ value is not dependent on the ambient parameters, and the approximation is valid to a few parts in $10^{3}$, neglecting the water vapor contribution.

On the other hand, the residual phase in the two wavebands $\sigma_{1}$ and $\sigma_{2}$ is, with (17) and (20):

$\phi_{1,2}=2 \pi \sigma_{1} \sigma_{2}\left(\sigma_{1}+\sigma_{2}\right) \beta L_{\mathrm{del}}$.

The sensitivity to path length variations of $\phi_{1}+\phi_{2}$ is larger than the sensitivity of group delay measurement, the gain factor being $\left(\sigma_{1}+\sigma_{2}\right) /\left(\sigma_{1}-\sigma_{2}\right)$, that is about 7.5 for the $\left(K_{\mathrm{s}}, H\right)$ pair. Once coherencing is achieved, optical path delay tracking with phase locking in one or the other waveband could be used, in a more sensitive way than group delay tracking alone. But a model for the residual phase variations with air delay length is needed, so that the interest of phase locking has to be demonstrated with a real experiment.

Group delay tracking will operate only with a significant $S N R$ in each wavebands, say larger than 5 . The uncertainty in the phase difference will then be smaller than $0.2 \sqrt{2} \mathrm{rad}$, or about 16 degrees per individual measurement. With about 50 measurements per second, a 1 degree rms value, or $20 \mathrm{~nm}$ with the $\left(K_{\mathrm{s}}, H\right)$ pair, is reached after 5 seconds of observing time. Such a sensitivity to path length variations is considered to be good enough for conveniently averaging the atmospheric piston in single-field astrometry, the duration of each observing sequence being larger than a few 10 seconds. For dual-field astrometry with two bright sources, the sensitivity in differential atmospheric piston will be $\sqrt{2}$ times the previous figure, that is better than $10 \mathrm{~nm}$ in a $45 \mathrm{~s}$ observing sequence, or better than $0.1 \mathrm{mas} / \mathrm{sequence}$ with a $30-40 \mathrm{~m}$ baseline, which is also considered as a consistent sensitivity figure.

\section{Conclusion}

The astrometric performance of a ground-based optical interferometer with air-filled delay lines has been investigated. Group delay tracking with zeroing the residual phase difference between two bands has been proposed, and shown to be only slightly dependent on the ambient parameters. With group delay tracking only, we have shown that dual-field astrometry at a precision level of a few $10^{-10}$ rad (or better than 0.1 mas) per observing sequence of a minute or so is achievable with air-filled delay lines and observation in the near-IR range. The interferometer baseline should not exceed a few 10 meters, for astrometric baseline calibration to be performed without compensation glass plates.

Acknowledgements. The authors acknowledge very fruitful discussions with F. Cassaing, P. Dierich and P. Juncar. We are grateful to M. Colavita for his comments and suggestions. This study was partly supported by a special grant (Action Incitative) from Observatoire de Paris, which is greatly acknowledged.

\section{References}

Armstrong J.T., Mozurkewich D., Rickard L.J., et al., 1998, ApJ 496, 550

Colavita M.M., Shao M., Staelin D.H., 1987, App. Opt. 26, 4113

Colavita M.M., Shao M., Hines B.E., et al., 1994, SPIE 2200, 89

Colavita M.M., Wallace J.K., Hines B.E., et al., 1999, ApJ 501, 505

Gubler J., Tytler D., 1998, PASP 110, 738

Koehler B., Lévêque S., von der Lühe O., 1997, SPIE 2871, 544

Lévêque S., 1997, "Méthodes et systèmes Laser pour l'analyse $\mathrm{du}$ trajet optique interne du très grand télescope interférométrique européen, VLTI", Thèse, Univ. de Strasbourg

Lawson P.R., 1995, J. Opt. Soc. Am. A 12, 366

Lawson P.R., Balwin J.E., Warner P.J., 1998, SPIE 3350, 753

Lawson P.R., Scott T.R., Haniff C.A., 1999, MNRAS 304, 218

Mariotti J.-M., Denise C., Derié F., et al., 1998, SPIE 3350, 800

Owens J.C., 1967, App. Opt. 6, 51 
Quirrenbach A., Coudé du Foresto V., Daigne G., et al., 1998, SPIE 3350, 807

Shao M., Staelin D.H., 1977, J. Opt. Soc. Am. 67, 81

Shao M., Staelin D.H., 1980, App. Opt. 19, 1519

Shao M., Colavita M.M., Hines B.E., et al., 1988, A\&A 193,
357

Shao M., Colavita M.M., 1992, A\&A 262, 353

Tango W.J., Twiss R.Q., 1980, Prog. Opt. XVII, 239

von der Lühe O., Ferrand D., Koehler B., et al., 1994, SPIE 2200,168 